\title{
A variational approach to a quasi-static droplet model
}

\author{
Natalie Grunewald • Inwon Kim
}

Received: 15 September 2009 / Accepted: 10 June 2010 / Published online: 8 July 2010

(C) The Author(s) 2010. This article is published with open access at Springerlink.com

\begin{abstract}
We consider a quasi-static droplet motion based on contact angle dynamics on a planar surface. We derive a natural time-discretization and prove the existence of a weak global-in-time solution in the continuum limit. The time discrete interface motion is described in comparison with barrier functions, which are classical sub- and super-solutions in a local neighborhood. This barrier property is different from standard viscosity solutions since there is no comparison principle for our problem. In the continuum limit the barrier properties still hold in a modified sense.
\end{abstract}

\section{Mathematics Subject Classification (2000) $\quad 35 \mathrm{~K} 55 \cdot 35 \mathrm{Q} 35 \cdot 35 \mathrm{~A} 01$}

\section{Introduction}

The motion of liquid drops on a planar surface is a widely studied topic. We consider a quasistationary free boundary model, derived in $[7,9,10]$. The model is contact angle driven, i.e. the motion of the boundary of the wetted region is due to a deviation of the contact angle from the ideal contact angle. It is also quasi-stationary in the sense that the actual profile of the drop adjusts itself to the wetted region by minimizing a "surface energy" under a volume constraint.

We derive a natural time discretization by exploiting a formal gradient flow structure of the model. The time-discrete solutions satisfy barrier properties similar to standard viscosity solutions. These barrier properties stay valid in a modified sense as the time step size goes to zero.

Let us begin by a formal introduction of the model. The profile of the droplet is given by the height function $u: \mathbb{R}^{N} \times(0, T) \rightarrow \mathbb{R}$ with $N=2$, the positive phase $\{u>0\}$ denotes the

Communicated by L. Ambrosio.

N. Grunewald ( $\square)$

Inst. für Angewandte Mathematik, Universität Bonn, Bonn, Germany

e-mail: grunewald@iam.uni-bonn.de

I. Kim

Department of Mathematics, UCLA, Los Angeles, CA 90095, USA

e-mail: ikim@math.ucla.edu 
wetted region and the free boundary $\partial\{u>0\}$ denotes the contact line between drop, air and surface. It should be pointed out that our analysis is performed in general space dimension $N$. Throughout the paper we denote the spatial derivative of $u$ by $D u$.

The motion of the droplet is described by contact angle dynamics - the free boundary $\partial\{u>0\}$ evolves by a relationship between the outward normal velocity $V$ and the contact angle $|D u|$ of the droplet with the surface. In this paper the normal velocity is given by

$$
V=|D u|^{2}-1 \quad \text { on } \partial\{u>0\} .
$$

The square of the contact angle in the velocity law seems natural, as it is the only power for which we directly have a gradient flow structure like the one considered in this paper. For discussion of the contact angle dynamics in form of more general free boundary velocities we refer to [15].

On the other hand the shape of the drop adjusts to the wetted region by obeying two constraints: First the volume in each component $\omega_{i}$ of the drop is kept constant over time. Secondly the liquid/vapor interface is minimal in the sense that it minimizes the Dirichlet integral, leading to the Euler-Lagrange equation

$$
-\Delta u(\cdot, t) \equiv \lambda
$$

in each connected components of $\{u>0\}$. This equation, a simplification of the minimal surface equation, defines the shape of a quasi-static droplet. By choosing a suitable Lagrange multiplier $\lambda=\lambda_{I}(x, t)$, the volume of droplets in each component can be preserved.

Summarizing above discussion we arrive at the following free boundary problem:

$$
(P) \begin{cases}-\Delta u(\cdot, t)=\lambda_{i}(t) \quad \text { in } \omega_{i}(t) ; \\ V=|D u|^{2}-1 & \text { on } \partial \omega_{i}(t) ; \\ \int_{\omega_{i}(t)} u(\cdot, t) d x \equiv c_{i}, & \end{cases}
$$

where, as mentioned above, $V$ is the outward normal velocity of the connected component of the support of the drop $\omega_{i}(t)$, so for $|D u| \neq 0$ one has $V=\frac{\partial_{t} u}{|D u|}$. As the overall volume is conserved we have $\sum_{i} c_{i} \equiv 1$.

Several serious challenges arise in developing a global notion of solutions for the model described above:

Most importantly, $(P)$ does not satisfy the comparison principle between solutions, even in the case of single components. For example consider two sets $D_{1} \subset D_{2} \subset \mathbb{R}^{n}$ with the droplet profile $u_{i}(x, 0)$ supported in $D_{i}$ for $i=1,2$. Suppose we have the same volume constraint, i.e., $\int u_{i}=1$. Since we assume a quasi-stationary profile for $u_{i}$, they satisfy the first equation in $(P)$ :

$$
-\Delta u_{i}(x, 0)=\lambda_{i} \text { in } D_{i},
$$

Due to the volume constraint and the fact that $D_{1} \subset D_{2}$, it is clear that $\lambda_{1}>\lambda_{2}$. Therefore it may be the case that $u_{1}>u_{2}$ in some parts of $D_{1}$. Also the fact that $\lambda_{1}>\lambda_{2}$ and the second equation in $(P)$ suggests the possibility that the free boundary velocity of $\left\{u_{1}>0\right\}$ is bigger than in $\left\{u_{2}>0\right\}$ in some parts, and therefore the evolution of $D_{1}$ and $D_{2}$ by $(P)$ may reverse the inclusion order between the sets.

Due to the failure of comparison principle, the viscosity solutions approach applied to mean curvature flow (see [3,6] for example) does not apply here, even if we assume that there is no topology change. Observe that if $\lambda$ is independent of time then standard viscosity solution theory as in [12] applies. Based on this observation a discrete-time approximation with fixed $\lambda$ in each time step was carried out in [8]. This way a unique weak solution is 
obtained for star-shaped initial data, for short times (as long as the wet region stays starshaped). However approximating $(P)$ with fixed $\lambda$ in small time intervals (apparently) does not work well with topology changes.

On the other hand topology changes seem unavoidable. Splitting of droplets into multiple components is generic for non-convex droplets, even if we start the evolution with a simply connected droplet. Merging of different parts of the droplet also naturally occurs. (Recall that our model is quasi-stationary. This means that the dynamics inside the liquid phase is not modeled. In some sense when a topology change occurs we "fast forward" the time so that the droplet becomes quasi-stationary again.) In addition to the topological changes, we expect corner or cusp formation on the interface, due to merging, splitting, and also shrinking of droplets (see [8]).

Lastly, there is a bifurcation (non-uniqueness) of solutions in the event of merging. More precisely, two stationary drops touching each other at exactly one point can either decide to stay as they are, or see each other and develop into one big drop. A similar bifurcation was also observed, for solutions of a flame propagation model [14].

Our goal is to introduce a global-time notion of weak solution which describes $(P)$ past topological changes and singularities. We take a variational approach, based on the following observation. Formally speaking the droplet evolution $(P)$ is a gradient flow for the energy

$$
E(\omega):=\int_{\omega}\left|D u_{\omega}\right|^{2} d x+|\omega|,
$$

where $|\omega|$ denotes the (Lebesgue) measure of $\omega$. The gradient flow takes place on the manifold of possible supports of the droplet. The droplet height $u$ itself is then part of a tangent bundle above the manifold. We refer to Sect. 2 for a detailed discussion of this structure. The energy $(P)$ is a linearized version of the surface energy

$$
E(\omega):=\int_{\omega} \sqrt{1+\left|D u_{\omega}\right|^{2}} d x+|\omega| .
$$

The linearization corresponds to the linear equation in the interior of problem $(P)$. Choosing the surface energy insteadt, would not only change the interior equation in $(P)$ to the minimal surface equation but also change the boundary conditions. This significantly changes the problem. Nevertheless, the methods introduced here might still be applicable.

In Sect. 2 we approximate the solution $(P)$ by a time-discrete gradient flow (JKO) scheme, originated by [11]. This scheme defines the solution in the next time step as a minimizer of a composited functional. This functional consists partly of the energy and partly of the distance to the previous time step. See Sect. 2 for details. Such approach was taken before by Almgren et al. [1] and Luckhaus and Sturzenhecker [13] for mean curvature motion. In [3] it was shown that a particular selection of the discrete scheme in [1] converges to viscosity solution of the mean curvature flow in the sense of [6]. Also see [2] where one studies a free boundary problem similar to $(P)$, but satisfies the comparison principle. (In [2] the goal was to obtain an energy bound for the viscosity solution of the corresponding problem.)

As mentioned above our problem lacks the comparison property even in simple settings, which prevents us to develop any connection to standard viscosity solutions approach. However it is still possible to describe the evolution of solutions by barrier properties (Propositions 3.1 and 3.3) of the time-discrete weak solutions. Roughly speaking this means that the time-discrete solutions evolve with the free boundary velocity given by $(P)$, at "regular" points of the interface. 
In the continuum limit we show that a global-in-time weak solution (see Definition 4.4) exists. At the moment, we are only able to describe the limiting free boundary behavior in terms of the liminf and limsup of the time-discrete solutions. We refer to Sect. 4 for definition of weak solutions (Definition 4.4) and precise statements (Theorem 4.6).

\section{Construction of a time discrete solution}

We consider a generalized version of $(P)$ with curvature:

$$
\left(P_{\epsilon}\right) \begin{cases}-\Delta u(\cdot, t)=\lambda_{i}(t) & \text { in } \omega_{i}(t) \\ V=|D u|^{2}-1-\epsilon \kappa & \text { on } \partial \omega_{i}(t) \\ \int_{\omega_{i}(t)} u(\cdot, t) d x \equiv \int u(x, 0) d x, & \end{cases}
$$

where $\omega_{i}$ is any connected component of supp $u$ and $\epsilon \geq 0$ with $\kappa=-\nabla \cdot\left(\frac{D u}{|D u|}\right)$ denotes the mean curvature of the interface, positive if the positive phase $\{u(\cdot, t)>0\}$ is convex. The curvature term in $(P)_{\epsilon}$ is introduced to use the structure of Caccioppoli sets in the variational arguments in Sect. 3. However the regularized problem $(P)_{\epsilon}$ and their properties are also of independent interest.

Let us start with the definitions:

Definition 2.1 Let $B:=\left\{x \in \mathbb{R}^{n}:|x|<R\right\}$ with $R$ a sufficiently large constant.

(a) Let us define the set of Caccioppoli sets

$$
\text { Cacc }:=\{\omega \subset B ; \omega \text { is a Borel set with finite perimeter }\} .
$$

(b) For any $\omega \in$ Cacc and any volume $c$

$$
u_{\omega, c}:=\operatorname{argmin}\left\{\int_{\omega}|D u|^{2} d x: \quad u \in H^{1}(\omega), \quad \operatorname{supp} u \subseteq \omega, \quad \int_{\omega} u d x=c\right\} .
$$

Remark 2.2 Note that the minimizer $u_{\omega, c}$ exists for any $c>0$ and any set $\omega \in$ Cacc that admits one $H^{1}$-function $u$ with supp $u \subseteq \omega$.

Definition 2.3 For a nonnegative function $u \in H^{1}\left(\mathbb{R}^{n}\right)$ and for $x \in \Omega(u)$ we define

$$
\lambda(u)(x):=\frac{\int_{\omega}|D u|^{2} d x}{\int_{\omega} u d x}
$$

where $\omega$ is the connected component of $\Omega(u)$ which contains $x$. If $\int_{\omega} u d x=0$ we set $\lambda(u)(x)=0$.

Note that $-\Delta u_{\omega, c}=\lambda\left(u_{\omega, c}\right)(x) \equiv \lambda\left(u_{\omega, c}\right)$ in its positive set, if $\omega$ has a single component with smooth boundary.

Problem $\left(P_{\epsilon}\right)$ is a formal gradient flow on Cacc for the energy

$$
E_{\epsilon}(\omega):=\int_{\omega}\left|D u_{\omega, 1}\right|^{2} d x+|\omega|+\epsilon \operatorname{per}(\omega),
$$

where $|\omega|$ and $\operatorname{per}(\omega)$ respectively denote the Lebesgue measure and the perimeter of $\omega$. To see this we calculate the differential of $E_{\epsilon}$ for some normal velocity field $\tilde{v}$ applied to $\partial \omega$ and $\delta \tilde{u}$ the change of $u$ introduced by $\tilde{v}$ : 


$$
\begin{aligned}
\operatorname{diff} E_{\epsilon}(\omega) . \tilde{v} & =\int_{\omega} 2 D u_{\omega} \cdot D \delta \tilde{u} d x+\int_{\partial \omega}\left(1+\left|D u_{\omega}\right|^{2}\right) \tilde{v} d S+\int_{\partial \omega} \epsilon \kappa \tilde{v} d S \\
& =-\int_{\omega} 2 \Delta u_{\omega} \delta \tilde{u} d x+\int_{\partial \omega}-2\left|D u_{\omega}\right| \delta \tilde{u}+\left(1+\left|D u_{\omega}\right|^{2}+\epsilon \kappa\right) \tilde{v} d S \\
& =\lambda \int_{\omega} \delta \tilde{u} d x+\int_{\partial \omega}-2\left|D u_{\omega}\right|^{2} \tilde{v}+\left(1+\left|D u_{\omega}\right|^{2}+\epsilon \kappa\right) \tilde{v} d S \\
& =\int_{\partial \omega}\left(1-\left|D u_{\omega}\right|^{2}+\epsilon \kappa\right) \tilde{v} d S .
\end{aligned}
$$

This gives $\left(P_{\epsilon}\right)$ for the Riemanian structure

$$
g_{\omega}(v, \tilde{v}):=\int_{\partial \omega} v \tilde{v} d S \quad \forall v, \tilde{v} \in T_{\omega} \text { Cacc },
$$

on $C a c c$, by the volume conservation and Definition 2.1. As the distance connected to (2.3) are difficult to model, we introduce a modified "distance", which was originally introduced in $[1,13]$ (also see e.g. $[3,4]$ )

$$
\widetilde{\operatorname{dist}}^{2}\left(\omega_{0}, \omega_{1}\right):=\int_{\omega_{0} \Delta \omega_{1}} \operatorname{dist}\left(x, \partial \omega_{0}\right) d x .
$$

Here dist is the distance function, and $\omega_{0} \Delta \omega_{1}$ denotes the symmetric difference between the two sets. Note that $\widetilde{d i s t}^{2}$ is not a (squared) distance function (it lacks e. g. symmetry), but an approximation of the distance connected to (2.3).

Following [13] and the JKO-scheme [11], $\omega_{h}^{i+1}$ is determined from the previous set $\omega_{h}^{i}$ by

$$
\omega_{h}^{i+1}=\underset{\omega \in \operatorname{Cacc}}{\operatorname{argmin}}\left\{\frac{1}{h} \widetilde{\operatorname{dist}}^{2}\left(\omega_{h}^{i}, \omega\right)+E_{\epsilon}(\omega)\right\} .
$$

Lemma 2.4 For fixed $h>0$, fixed volume $c$ and any $\omega^{0} \in$ Cacc there exists at least one minimizer $\omega_{c}^{\min } \in$ Cacc of

$$
\mathcal{F}(\omega):=\frac{1}{h} \widetilde{\operatorname{dist}}^{2}\left(\omega^{0}, \omega\right)+E_{\epsilon}(\omega) .
$$

Note that we do not show uniqueness. We also do not expect uniqueness for $(P)$ or $\left(P_{\epsilon}\right)$, see Sect. 1. The dependence on $c$ is suppressed in the notation of $E_{\epsilon}$.

Proof There exist sets $\omega \subset B$ such that $\mathcal{F}(\omega)<\infty$ (e.g. spheres around $\left.\omega^{0}\right)$ and $\mathcal{F}(\omega) \geq 0$ for all $\omega$. Therefore there exists a minimizing sequence $\left\{\omega_{k}\right\} \subset$ Cacc such that

$$
\mathcal{F}\left(\omega_{k}\right) \underset{k \rightarrow \infty}{\rightarrow} \inf \{\mathcal{F}(\omega): \quad \omega \subset B\} .
$$

By the definition of $E_{\epsilon}(\omega)$ we have $\left|\omega_{k}\right|+\epsilon \operatorname{per}\left(\omega_{k}\right)<C$ and therefore the indicator functions $\chi_{\omega_{k}}$ are uniformly bounded in BV-norm. Thus (see e.g. [5], p. 176) there exists a subsequence and a function $\chi \in B V(B)$ such that

$$
\chi_{\omega_{k}} \rightarrow \chi \quad \text { in } L^{1}(B)
$$

Since $\chi_{\omega_{k}}$ take values in $\{0,1\}$ so does $\chi$ and there exists a set $\omega_{c}^{\min } \subset B$ such that $\chi=\chi_{\omega_{c}^{\min }}$. 
It remains to show that $\mathcal{F}\left(\omega_{c}^{\text {min }}\right) \leq \inf \mathcal{F}\left(\omega_{k}\right)$. This is direct for the part of the energy $\left|\omega_{k}\right|+\epsilon \operatorname{per}\left(\omega_{k}\right)$, by the lower semi continuity of the perimeter and the $L^{1}$ convergence of $\chi_{\omega_{k}}$. For the remaining part of the energy we have to take into account the convergence of the corresponding droplet with volume $c, u_{\omega_{k}, c}$. By the boundedness of the $H^{1}$-norm of $u_{\omega_{k}, c}$

$$
u_{\omega_{k}, c} \rightarrow \tilde{u} \quad \text { in } L^{2}(B) .
$$

where $\int \tilde{u}=c$ and

$$
u_{\omega_{k}, c}=u_{\omega_{k}, c} \chi_{\omega_{k}} \rightarrow \tilde{u} \chi_{\omega_{c}^{\min }} \text { a.e. in } B .
$$

Therefore by the lower semi-continuity of $H^{1}$-norm and Definition 2.1

$$
\inf \int_{\omega_{k}, c}\left|D u_{\omega_{k}, c}\right|^{2} \geq \int_{\omega_{c}^{\min }}|D \tilde{u}|^{2} \geq \int_{\omega_{c}^{\min }}\left|D u_{\omega^{\min }, c}\right|^{2} .
$$

On the other hand $\widetilde{d i s t}^{2}$ is continuous with respect to the $L^{1}$-topology of the indicator functions:

$$
\begin{aligned}
\left|\widetilde{\operatorname{dist}}^{2}\left(\omega^{0}, \omega\right)-\widetilde{\operatorname{dist}}^{2}\left(\omega^{0}, \bar{\omega}\right)\right| & =\left|\int_{\omega^{0} \Delta \omega} \operatorname{dist}\left(x, \partial \omega^{0}\right) d x-\int_{\omega^{0} \Delta \bar{\omega}} \operatorname{dist}\left(x, \partial \omega^{0}\right) d x\right| \\
& =\left|\int_{(\omega \Delta \bar{\omega}) \Delta \omega^{0}} \operatorname{dist}\left(x, \partial \omega^{0}\right) d x\right| .
\end{aligned}
$$

This vanishes as $\left\|\chi_{\omega}-\chi_{\bar{\omega}}\right\|_{L^{1}\left(\mathbb{R}^{N}\right)}=|\omega \Delta \bar{\omega}| \rightarrow 0$, by the boundedness of the distance function in $B$.

\subsection{Definition of the time-discrete evolution}

We define a time-discrete evolution of $(P)$. Roughly speaking we do the minimization in Lemma 2.4 for each component of the drop separately. If two components merge at the next time step, we go back and do the same minimization step but for the two components together. Splitting of a component is already taken care of in the minimization in Lemma 2.4, as $\omega^{\text {min }}$ might have several components. To be more precise: for fixed $h>0$ and $i \in \mathbb{N}$ take the previous state $\omega_{h}^{i} \in C a c c$ with possibly infinitely many connected components $\omega_{h}^{i, k} \in C a c c, k \in \mathbb{N}$. For each connected component (in the classical sense) we have some droplet $u_{\omega_{h}^{i, k}, c_{k}}$ by Remark 2.2 and Lemma 2.4. Then $\omega_{h}^{i+1}$ is given by

$$
\omega_{h}^{i+1}:=\bigcup_{k} \omega_{c_{k}}^{\min } \quad \text { if for any } l \neq m: \quad \omega_{c_{l}}^{\min } \cap \omega_{c_{m}}^{\min }=\emptyset,
$$

where $\omega_{c_{k}}^{\min }$ is a minimizer in Lemma 2.4 for the connected component $\omega_{h}^{i, k}$.

If $\omega_{c_{l}}^{\text {min }} \cap \omega_{c_{m}}^{\text {min }} \neq \varnothing$ for only one pair $(l, m)$ and if it does not intersect with other components $\left(\omega_{c_{k}}^{\min }, k \neq l, m\right)$, then we define

$$
\omega_{h}^{i+1}:=\left(\bigcup_{k \neq l, m} \omega_{c_{k}}^{\min }\right) \cup \omega_{c_{l}+c_{k}}^{\min } .
$$

where $\omega_{c_{l}+c_{k}}^{\min }$ is a minimizer in Lemma 2.4 for initial set $\omega_{h}^{i, l} \cup \omega_{h}^{i, m}$. 
In general the process of sorting out merging components is non-unique: we will prescribe the following process to proceed without ambiguity. Let us first consider the maximal index set $I_{1}$ such that each element $\omega_{c_{k}}^{\min }$ with $k \in I_{1}$ intersects with $\omega_{c_{1}}^{\min }$.

Next take the first element $\omega_{c_{k}}^{\text {min }}$ with $k \notin I_{1}$ and repeat the process to create the second index set $I_{2}$. If $I_{2}$ intersects with $I_{1}$, then we replace $I_{1}$ with $I_{1} \cup I_{2}$. If not, check whether

$$
\omega_{I_{1}}^{\min }:=\omega_{\Sigma c_{k}}^{\min }, \quad k \in I_{1}
$$

intersects with $\omega_{I_{2}}^{\min }$. If yes then still replace $I_{1}$ with $I_{1} \cup I_{2}$. If no, then proceed to create the third index set $I_{3}$, and check against $\omega_{I_{1}}^{\text {min }}$ and $\omega_{I_{2}}^{\text {min }}$. This way we end up with a sequence of (disjoint) index sets $I_{1}, I_{2}, \ldots$ such that $\omega_{I_{k}}^{\min }$ are all disjoint. Then

$$
\omega_{h}^{i+1}:=\bigcup_{k} \omega_{I_{k}}^{\min }
$$

Now define

$$
u_{I_{k}}:=u_{\omega_{I_{k}}^{\min }, \Sigma_{j \in I_{k}} c_{j}}
$$

and

$$
u_{h}(\cdot, t):=\sum_{k} u_{I_{k}} \quad \text { for } t \in[i h,(i+1) h)
$$

This way $u_{h}$ is a $H^{1}$-function in $B$ at any time $t \in[0, T]$. Thus

$$
u_{h} \in L_{l o c}^{2}\left(H^{1}(B)\right) \text {. }
$$

The total volume of $u_{h}$ at time $t$ is $\int u_{h}(\cdot, t) d x=\sum_{k} c_{k}=1$.

As the JKO-scheme is constructed to describe a time-discrete gradient flow, we have the energy decrease for free: Suppressing in the notation the dependence of the energy on the volumes in each component, we have:

Lemma 2.5 The time evolution defined in (2.4) and (2.5) satisfies

$$
E_{\epsilon}\left(\omega_{h}^{i}\right) \geq \frac{1}{h} \widetilde{\operatorname{dist}}^{2}\left(\omega_{h}^{i}, \omega_{h}^{i+1}\right)+E_{\epsilon}\left(\omega_{h}^{i+1}\right) .
$$

Proof Equation (2.6) is obvious for any components $\bigcup_{k \in I_{j}} \omega_{h}^{i, k}$ and the corresponding minimizer $\omega_{I_{j}}^{\text {min }}=\omega_{I_{j}}^{i+1}$, as Lemma 2.4 can be tested with the set $\bigcup_{k \in I_{j}} \omega_{h}^{i, k}$. Furthermore

$$
\begin{aligned}
E_{\epsilon}\left(\omega_{h}^{i}\right)=\sum_{j} E_{\epsilon}\left(\bigcup_{k \in I_{j}} \omega_{h}^{i, k}\right) & \geq \frac{1}{h} \sum_{j} \widetilde{\operatorname{dist}}^{2}\left(\bigcup_{k \in I_{j}} \omega_{h}^{i, k}, \omega_{I_{j}}^{\min }\right)+\sum_{j} E_{\epsilon}\left(\omega_{I_{j}}^{\min }\right) \\
& \geq \frac{1}{h} \widetilde{\operatorname{dist}}^{2}\left(\omega_{h}^{i}, \omega_{h}^{i+1}\right)+E_{\epsilon}\left(\omega_{h}^{i+1}\right) .
\end{aligned}
$$

\section{The barrier properties for time discrete solutions}

In this section we show, that for fixed time step $h>0$ the discrete-time solution constructed above satisfies the free boundary motion law in time scale $h$, in the sense that it is comparable 
to smooth sub- and super-solutions of $\left(P_{\epsilon}\right)$ in local neighborhoods. A more precise statement will follow in Propositions 3.1 and 3.3 for which we need the following notation:

Let us denote the positive phase of a function $u(x, t): \mathbb{R}^{N} \times[0, \infty) \rightarrow \mathbb{R}^{+}$and its boundary by:

$$
\Omega_{t}(u):=\{u(\cdot, t)>0\} \quad \text { and } \quad \Gamma_{t}(u):=\partial\{u(\cdot, t)>0\},
$$

and the positive phase in space-time by:

$$
\Omega(u):=\{u>0\} \subset\{B \times[0, \infty)\} \quad \text { and } \quad \Gamma(u):=\partial \Omega(u) .
$$

Next we show the barrier properties for the time discrete solutions. We begin with the barrier property for $u_{h}$ being a super-solution. That is, $u_{h}$ can be compared to a barrier function $\phi$ that is below. If $\phi$ is not fast enough at the boundary and not curved enough in the interior, then the ordering will persist:

Proposition 3.1 (Super-solution barrier property) Let $u_{h}$ be defined by (2.5). Given a ball $B_{r}\left(x_{0}\right)$ in $B$ let

$$
\lambda:=\inf _{x \in B_{r}\left(x_{0}\right)}\left\{\lambda\left(u_{h}(0, \cdot)\right)(x), \lambda\left(u_{h}(h, \cdot)\right)(x)\right\},
$$

where $\lambda(u)(x)$ is as defined by (2.1).

Suppose there exists a smooth function $\phi$ with $|D \phi| \neq 0$ in $B_{r}\left(x_{0}\right) \times[0, h]$. Further suppose that for some small $\delta>0$

$$
\begin{array}{r}
-\Delta \phi(\cdot, t)<\lambda-\delta \quad \text { in } \quad B_{r}\left(x_{0}\right) \times[0, h], \\
\frac{\phi_{t}}{|D \phi|}-\left(|D \phi|^{2}-1-\epsilon \kappa_{\phi}\right)<-\delta \quad \text { on } \quad \Gamma(\phi) \cap\left(B_{r}\left(x_{0}\right) \times[0, h]\right),
\end{array}
$$

where $\kappa_{\phi}:=-\nabla \cdot\left(\frac{D \phi}{|D \phi|}\right)$ is the mean curvature of the corresponding level set of $\phi$. Then for sufficiently small $h>0$-depending on $\delta, r$, the minimum of $|D \phi|$ and the $C^{2}$-norm of $\phi$ in $B_{r}\left(x_{0}\right) \times[0, h]$ - the following holds:

If $\phi \leq u_{h}$ on the parabolic boundary of $B_{r}\left(x_{0}\right) \times[0, h]$, then $\phi(\cdot, h) \leq u_{h}(\cdot, h)$ in $B_{r}\left(x_{0}\right)$.

Note that $\frac{\phi_{t}}{|D \phi|}=V$, where $V$ is the outward normal velocity of $\partial\{\phi>0\}$ with respect to the positive set of $\phi$. Therefore, Proposition 3.1 shows that a function $\phi$ which is a sub-solution of $\left(P_{\epsilon}\right)$ can not cross the discrete time solution $u_{h}$. Thus, $u_{h}$ is a super-solution. We also mention that a local barrier function like the ones in Proposition 3.1 can always be extended to a global barrier function satisfying (3.1), which is not restricted to a ball $B_{r}$.

We begin by a lemma which states that the support of $\phi$ cannot cross $\omega_{h}$ too much.

Lemma 3.2 Under the assumptions of Proposition 3.1 and for sufficiently small $h>0$, there exists a constant $C>0$ independent of $h>0$ such that

$$
(\phi(x, t)-C h)_{+} \leq u_{h}(x, t) \text { in } B_{r}\left(x_{0}\right) \times[0, h] .
$$

In particular, if $\phi$ crosses $u_{h}$ on $B_{r}\left(x_{0}\right) \times[0, h]$, we can choose $-C h<\tau<$ Ch such that $\varphi(x, t):=(\phi(x, t)-\tau)_{+}$crosses $\omega_{h}:=\Omega_{h}\left(u_{h}\right)$ from below by $o\left(h^{2}\right)$, i.e.,

$$
0<\left|\left(\Omega_{h}(\varphi)-\omega_{h}\right) \cap B_{r}\left(x_{0}\right)\right|=o\left(h^{2}\right) .
$$

Note, that we need the possibility of a negative $\tau$ to ensure for an intersection in the case where $\phi$ crosses $u_{h}$ between the times $0 \leq t \leq h$. 
Proof Once (3.2) is proved, our second claim follows from the fact that $\Omega_{h}(\phi)$ does cross $\omega_{h}$ and $\phi$ is smooth with $|D \phi|>0$ near $\Omega(\phi)$.

To prove (3.2), first observe that

$$
\Omega_{h}(\phi) \subset\left\{\Omega_{0}(\phi) \cup\left\{x \in B: d\left(x, \Gamma_{0}(\phi)\right) \leq C_{1} h\right\}\right\},
$$

where

$$
C_{1}=\sup _{B_{r}\left(x_{0}\right) \times[0, h]}\left|\phi_{t}\right| /|D \phi| .
$$

Thus, (3.2) follows by the comparison principle if we show

$$
S:=\left\{x \in\left(\Omega_{0}(\phi) \cap B_{r}\left(x_{0}\right)\right): d\left(x, \Gamma_{0}(\phi)\right) \geq C_{2} h\right\} \subset \omega_{h} .
$$

To prove (3.4), let us define

$$
\hat{\omega}_{h}:=\omega_{h} \cup S \quad \text { and } \quad \Sigma:=\hat{\omega}_{h}-\omega_{h}=S-\omega_{h} .
$$

Suppose that $|\Sigma| \neq 0$, otherwise we are done. Since $\Omega_{0}(\phi) \subset \omega_{0}$, we have by the smoothness of $\phi$

$$
\widetilde{\operatorname{dist}}^{2}\left(\omega_{0}, \hat{\omega}_{h}\right)-\widetilde{\operatorname{dist}}^{2}\left(\omega_{0}, \omega_{h}\right) \leq-C_{3} h|\Sigma| \text {. }
$$

where $C_{3}$ is proportional to the size of $C_{2}$. On the other hand, since the Dirichlet energy decreases when the domain increases,

$$
\begin{aligned}
E_{\epsilon}\left(\hat{\omega}_{h}\right)-E_{\epsilon}\left(\omega_{h}\right) & \leq|\Sigma|+\epsilon \operatorname{per}\left(\hat{\omega}_{h}\right)-\epsilon \operatorname{per}\left(\omega_{h}\right) \\
& \leq|\Sigma|+\epsilon\left|\partial S-\omega_{h}\right|-\epsilon\left|\partial \omega_{h} \cap S\right| \\
& \leq C_{4}|\Sigma|,
\end{aligned}
$$

where $C_{4}$ depends on $\phi$. The last inequality follows from

$$
\begin{aligned}
\left|\partial S-\omega_{h}\right|-\left|\partial \omega_{h} \cap S\right| & \leq \int_{\partial \Sigma}-\frac{D \phi}{|D \phi|}(x, h) \cdot \eta d S \\
& =-\int_{\Sigma} \nabla \cdot\left(\frac{D \phi}{|D \phi|}\right)(x, h) d x \\
& =\int_{\Sigma} \kappa_{\phi} d x .
\end{aligned}
$$

Here $\eta$ is the outward normal vector at $x \in \partial \Sigma$ and $\kappa_{\phi}$ is the mean curvature of the level set of $\phi$. We conclude that if $C_{3}$ is chosen sufficiently large, then

$$
\widetilde{\operatorname{dist}}^{2}\left(\omega_{0}, \hat{\omega}_{h}\right)+h E_{\epsilon}\left(\hat{\omega}_{h}\right)<\widetilde{\operatorname{dist}}^{2}\left(\omega_{0}, \omega_{h}\right)+h E_{\epsilon}\left(\omega_{h}\right) .
$$

This contradicts the minimizing property of $\omega_{h}$.

Proof of Proposition 3.1 (1) Suppose the proposition is not true. Then $\phi\left(x_{0}, h\right)>$ $u_{h}\left(x_{0}, h\right)$ at some point $x_{0} \in \Omega_{h}\left(u_{h}\right)$. Due to the maximum principle for harmonic functions, this implies that $\Omega_{h}(\phi) \cap\left(B \backslash \omega_{h}\right) \neq \varnothing$ for one of the components $\omega_{h}$ in $\Omega_{h}\left(u_{h}\right)$. Let $\omega_{0}$ be a corresponding component in $\Omega_{0}\left(u_{h}\right)$ which gives rise to $\omega_{h}$.

For notational simplicity, we prove the proposition assuming that $\omega_{h}$ is indeed the only component generated by $\omega_{0}$, i.e. $\omega_{0}$ has not splitted into multiple components and $\omega_{h}$ is generated by only one component: the proof for the general case is parallel. 
(2) Let us define

$$
\varphi(x, t):=(\phi(x, t)-\tau)_{+}
$$

where $-h^{2}<\tau<C h^{1 / 2}$ is as given in Lemma 3.2. In the proof we use $\varphi$ instead of $\phi$, which is possible without violating the assumptions according to Lemma 3.2. Next set

$$
\tilde{\omega}_{h}:=\omega_{h} \cup\left(\Omega_{h}(\varphi) \cap B_{r}\left(x_{0}\right)\right) .
$$

We claim that

$$
\widetilde{\operatorname{dist}}^{2}\left(\omega_{0}, \tilde{\omega}_{h}\right)+h E_{\epsilon}\left(\tilde{\omega}_{h}\right)<\widetilde{\operatorname{dist}}^{2}\left(\omega_{0}, \omega_{h}\right)+h E_{\epsilon}\left(\omega_{h}\right),
$$

which yields a contradiction to the minimizing property of $\omega_{h}$.

(3) To prove (3.5) first observe that

$$
\begin{aligned}
\widetilde{\operatorname{dist}}^{2}\left(\omega_{0}, \tilde{\omega}_{h}\right)-\widetilde{\operatorname{dist}}^{2}\left(\omega_{0}, \omega_{h}\right) & =\int_{\tilde{\omega}_{h} \Delta \omega_{h}} \operatorname{signdist}\left(x, \partial \omega_{0}\right) d x \\
& \leq \int_{\tilde{\omega}_{h} \Delta \omega_{h}} \operatorname{signdist}\left(x, \Gamma_{0}(\varphi)\right) d x,
\end{aligned}
$$

where signdist is the signed distance function, that is negative inside the set. Here the first equality is due to straightforward computation, and the inequality is due to the fact that $\Omega_{0}(\varphi)$ is a subset of $\omega_{0}$. By construction of $\varphi$, for each point $x \in \tilde{\omega}_{h} \Delta \omega_{h}$ there exists a time $t^{*}$ with $0 \leq t^{*} \leq h+o\left(h^{2}\right)$ such that $x \in \Gamma_{t^{*}}(\varphi)$. (The term $o\left(h^{2}\right)$ is added due to the possible negativeness of $\tau$.) Therefore, as $\frac{\varphi_{t}}{|D \varphi|}(0, \cdot)$ denotes the outward normal velocity of $\Gamma(\varphi)$,

$$
\operatorname{signdist}\left(x, \Gamma_{0}(\varphi)\right) \leq h \frac{\varphi_{t}}{|D \varphi|}+o(h) .
$$

Next we consider the energy difference

$$
E_{\epsilon}\left(\omega_{h}\right)-E_{\epsilon}\left(\tilde{\omega}_{h}\right)=I+I I+I I I
$$

where

$$
I=\int\left|D u_{h}\right|^{2}(\cdot, h)-\int\left|D \tilde{u}_{h}\right|^{2}, \quad I I=-\int_{\tilde{\omega}_{h} \Delta \omega_{h}} 1 d x, \quad I I I=\epsilon \operatorname{per}\left(\omega_{h}\right)-\epsilon \operatorname{per}\left(\tilde{\omega}_{h}\right) .
$$

Here $\tilde{u}_{h}(x):=u_{\tilde{\omega}_{h}, \int u_{h}}$. In the next step we will show that

$$
I \geq \int_{\tilde{\omega}_{h} \Delta \omega_{h}}|D \varphi|^{2}(\cdot, h) d x \quad \text { and } \quad I I I \geq \int_{\tilde{\omega}_{h} \Delta \omega_{h}}-\epsilon \kappa_{\varphi} d x
$$

This proves our claim by (3.1) and (3.6). Note that (3.1) is strict and therefore extends to a small region inside. 
(4) Let us estimate $I I I$. Note that, as before,

$$
\begin{aligned}
\operatorname{per}\left(\omega_{h}\right)-\operatorname{per}\left(\tilde{\omega}_{h}\right) & \geq \int_{\partial \omega_{h} \backslash \partial \tilde{\omega}_{h}}-\frac{D \varphi}{|D \varphi|}(\cdot, h) \cdot \eta d S-\int_{\partial \tilde{\omega}_{h} \backslash \partial \omega_{h}}-\frac{D \varphi}{|D \varphi|}(\cdot, h) \cdot \tilde{\eta} d S \\
& =\int_{\tilde{\omega}_{h} \Delta \omega_{h}} \nabla \cdot\left(\frac{D \varphi}{|D \varphi|}\right)(\cdot, h) d x \\
& =\int_{\tilde{\omega}_{h} \Delta \omega_{h}}-\kappa_{\varphi} d x
\end{aligned}
$$

where $\tilde{\eta}=-D \varphi /|D \varphi|(x, h)$ is the outward normal vector at $x \in \partial \tilde{\omega}_{h}, \eta$ is the outward normal vector at $x \in \partial \omega_{h}$, and $\kappa_{\varphi}$ is the mean curvature of the level sets of $\varphi$.

It remains to estimate $I$. To this end let us define two auxiliary functions, $\bar{u}$ and $v$ :

$$
\begin{array}{ll}
-\Delta \bar{u}=\lambda \quad \text { in } \tilde{\omega}_{h} \text { with } \quad \operatorname{supp}(\bar{u})=\overline{\tilde{\omega}}_{h}, \\
-\Delta v=0 \quad \text { in } \omega_{h} \text { with } \quad v=\varphi(\cdot, h) \text { on } \partial \omega_{h} .
\end{array}
$$

We remark that $\bar{u}$ is defined by approximation from outside and $v$ is defined by approximation from inside, i.e.

$$
\bar{u}(x):=\inf \left\{f(x):-\Delta f=\lambda \text { in }\{f>0\} \text { with } \tilde{w}_{h} \subset \overline{\{f>0\}}\right\}
$$

and

$$
v(x):=\sup \left\{f(x):-\Delta f=0 \text { in } \omega_{h} \text { with } f<\phi \text { on } B \backslash \omega_{h}\right\} .
$$

Let us define $c:=\int_{\omega_{h}} u_{h}(\cdot, h), \bar{c}:=\int_{\tilde{\omega}_{h}} \bar{u}$ and $\tilde{u}:=\frac{c}{\bar{c}} \bar{u}$. Then the following holds:

$$
\begin{aligned}
\int_{\omega_{h}}\left|D u_{h}(\cdot, h)\right|^{2}-\int_{\tilde{\omega}_{h}}\left|D \tilde{u}_{h}\right|^{2} & =\lambda \int_{\omega_{h}} u_{h}(\cdot, h)-\lambda(\tilde{u}) \int_{\tilde{\omega}_{h}} \tilde{u} \\
& =\frac{c}{\bar{c}} \lambda\left(\int_{\tilde{\omega}_{h}} \bar{u}-\int_{\omega_{h}} u_{h}\right) .
\end{aligned}
$$

Furthermore, $\bar{u} \geq \max \left(\left.\left(u_{h}+v\right)\right|_{\omega_{h}}, \varphi\right)(\cdot, h)$ since

$$
\tilde{\omega}_{h}=\Omega_{h}\left(\max \left(\left.\left(u_{h}+v\right)\right|_{\omega_{h}}, \varphi\right)\right) \text { and } \max \left(-\left.\Delta\left(u_{h}+v\right)\right|_{\omega_{h}},-\Delta \varphi\right)(\cdot, h) \leq \lambda \text {. }
$$

For the same reason, on the reduced boundary of $\omega_{h}$ we have, for the inward normal $\eta$,

$$
\partial_{\eta}\left(u_{h}(\cdot, h)+v\right) \geq \partial_{\eta} \varphi(\cdot, h) .
$$


Thus,

$$
\begin{aligned}
\lambda\left(\int_{\tilde{\omega}_{h}} \bar{u}-\int_{\omega_{h}} u_{h}(\cdot, h) \geq\right. & \lambda \int_{\omega_{h}} v+\lambda \int_{\omega_{h} \Delta \tilde{\omega}_{h}} \varphi(\cdot, h) \\
\geq & -\int_{\omega_{h}} \Delta\left(u_{h}(\cdot, h)+v\right) v-\int_{\omega_{h} \Delta \tilde{\omega}_{h}}(\Delta \varphi \varphi)(\cdot, h) \\
\geq & \int_{\omega_{h}}\left(D\left(u_{h}(\cdot, h)+v\right)\right) D v+\int_{\partial \omega_{h}} \partial_{\eta}\left(u_{h}(\cdot, h)+v\right) v \\
& +\int_{\omega_{h} \Delta \tilde{\omega}_{h}}|D \varphi|^{2}(\cdot, h)-\int\left(\partial_{\eta} \varphi \varphi\right)(\cdot, h) \\
\geq & \int_{\omega_{h}}|D v|^{2}+\int_{\omega_{h} \Delta \tilde{\omega}_{h}}|D \varphi|^{2}(\cdot, h),
\end{aligned}
$$

by (3.9) and (3.7). Thus together with (3.8) we have

$$
I \geq \frac{c}{\bar{c}} \int_{\omega_{h} \Delta \tilde{\omega}_{h}}|D \varphi|^{2}(\cdot, h) .
$$

Lastly, note that due to (3.3) $\lambda(\tilde{u})$ converges to $\lambda\left(u_{h}\right)$, as $h \rightarrow 0$. Hence $\bar{c} \rightarrow c$ and we can choose $h$ small enough that $\bar{c} \leq c(1+O(h))$ to conclude.

By a parallel argument in the proof of Proposition 3.1, $u_{h}$ can also be compared with barriers which are super-solutions of $\left(P_{\epsilon}\right)$ :

Proposition 3.3 (Sub-solution: barrier property) Let $u_{h}$ be defined by (2.5). Given a ball $B_{r}\left(x_{0}\right)$ in $B$, let

$$
\lambda:=\inf _{x \in B_{r}\left(x_{0}\right)}\left\{\lambda\left(u_{h}(0, \cdot)\right)(x), \lambda\left(u_{h}(h, \cdot)\right)(x)\right\},
$$

where $\lambda(u)(x)$ is as defined in (2.1).

Suppose there exists a smooth function $\phi$ with $|D \phi| \neq 0$ in $B_{r}\left(x_{0}\right) \times[0, h]$. Further suppose that for some small $\delta>0$

$$
-\Delta \phi(\cdot, t)>\lambda+\delta \quad \text { and } \quad \frac{\phi_{t}}{|D \phi|}-\left(|D \phi|^{2}-1-\epsilon \kappa_{\phi}\right)>\delta \quad \text { in } B_{r}\left(x_{0}\right) \times[0, h] .
$$

Then for sufficiently small $h>0$-depending on $\delta, r$, the minimum of $|D \phi|$ and the $C^{2}$-norm of $\phi$ in $B_{r}\left(x_{0}\right) \times[0, h]-$ the following holds:

If $u_{h} \leq \phi_{+}:=\max (\phi, 0)$ on the parabolic boundary of $B_{r}\left(x_{0}\right) \times[0, h]$, then $u_{h}(\cdot, h) \leq$ $\phi(\cdot, h)_{+}$in $B_{r}\left(x_{0}\right)$.

Proof The proof is analogous to the proof of Proposition 3.1. We still present it, as the estimation of the Dirichlet integral has a non-trivial difference from the previous proof.

Suppose the above proposition is not true. Then $\phi(\cdot, h)$ crosses $u_{h}(\cdot, h)$ from above at some point in $B_{r}\left(x_{0}\right)$. As before, the maximum principle for harmonic functions states that then $\Omega_{h}(\phi) \cap \omega_{h}$ is nonempty for a component $\omega_{h}$ of $\Omega_{h}\left(u_{h}\right)$. Set $\omega_{0}$ be the component of 
$\Omega_{0}\left(u_{h}\right)$ which generates $\omega_{h}$. Again we construct a contradiction to the minimizing property of $\omega_{h}$ and $u_{h}$. With a parallel argument to Lemma 3.2 one can change $\phi$ to $\varphi:=(\phi+\tau)_{+}$, $h^{2} \leq \tau \leq C h^{1 / 2}$, such that $u_{h}(x, h) \leq(\phi(x, h)+\tau)_{+}$and

$$
0<\left|\left(\omega_{h}-\Omega_{h}(\varphi)\right) \cap B_{r}\left(x_{0}\right)\right|=o\left(h^{2}\right) .
$$

This time we denote:

$$
\tilde{\omega}_{h}=\left(\left(\omega_{h} \cap \Omega_{h}(\varphi)\right) \cap B_{r}\left(x_{0}\right)\right) \cup\left(\omega_{h} \cap\left(B \backslash B_{r}\left(x_{0}\right)\right) .\right.
$$

We claim that

$$
\widetilde{\operatorname{dist}}^{2}\left(\omega_{0}, \tilde{\omega}_{h}\right)+h E_{\epsilon}\left(\tilde{\omega}_{h}\right)<\widetilde{\operatorname{dist}}^{2}\left(\omega_{0}, \omega_{h}\right)+h E_{\epsilon}\left(\omega_{h}\right) .
$$

First observe that this time

$$
\begin{aligned}
\widetilde{\operatorname{dist}}^{2}\left(\omega_{0}, \tilde{\omega}_{h}\right)-\widetilde{\operatorname{dist}}^{2}\left(\omega_{0}, \omega_{h}\right) & =-\int_{\tilde{\omega}_{h} \Delta \omega_{h}} \operatorname{signdist}\left(x, \partial \omega_{0}\right) d x \\
& \leq-\int_{\tilde{\omega}_{h} \Delta \omega_{h}} \operatorname{signdist}\left(x, \Gamma_{0}(\varphi)\right) d x .
\end{aligned}
$$

By integration of the velocity of $\Gamma_{t}(\varphi)$ we have

$$
-\operatorname{signdist}\left(x, \Gamma_{0}(\varphi)\right) \leq-h \frac{\varphi_{t}}{|D \varphi|}+o(h) .
$$

Next we consider the energy difference

$$
E_{\epsilon}\left(\omega_{h}\right)-E_{\epsilon}\left(\tilde{\omega}_{h}\right)=I+I I+I I I
$$

where

$$
I=\int\left|D u_{h}\right|^{2}(\cdot, h)-\int\left|D \tilde{u}_{h}\right|^{2}, \quad I I=\int_{\tilde{\omega}_{h} \Delta \omega_{h}} 1 d x, \quad I I I=\epsilon \operatorname{per}\left(\omega_{h}\right)-\epsilon \operatorname{per}\left(\tilde{\omega}_{h}\right) .
$$

Here $\tilde{u}(x)$ solves $-\Delta \tilde{u}=\tilde{\lambda}$ with support $\tilde{\omega}_{h}$, where $\tilde{\lambda}$ is chosen such that $\int \tilde{u}=\int u_{h}(\cdot, h)$. We will show that

$$
I \geq-\int_{\tilde{\omega}_{h} \Delta \omega_{h}}|D \varphi|^{2}(\cdot, h) d x \quad \text { and } \quad I I I \geq \int_{\tilde{\omega}_{h} \Delta \omega_{h}} \epsilon \kappa_{\varphi} d x
$$

This proves our claim by (3.10), (3.12) and (3.11).

First let us estimate $I I I$ :

$$
\begin{aligned}
\operatorname{per}\left(\omega_{h}\right)-\operatorname{per}\left(\tilde{\omega}_{h}\right) & \geq \int_{\partial \omega_{h} \backslash \partial \tilde{\omega}_{h}} \frac{D \varphi}{|D \varphi|}(\cdot, h) \cdot \eta d S-\int_{\partial \tilde{\omega}_{h} \backslash \partial \omega_{h}} \frac{D \varphi}{|D \varphi|}(\cdot, h) \cdot \tilde{\eta} d S \\
& =-\int_{\tilde{\omega}_{h} \Delta \omega_{h}} \nabla \cdot\left(\frac{D \varphi}{|D \varphi|}\right)(\cdot, h) d x \\
& =\int_{\tilde{\omega}_{h} \Delta \omega_{h}} \kappa_{\varphi} d x
\end{aligned}
$$


where $\tilde{\eta}=-D \varphi /|D \varphi|(x, h)$ is the outward normal vector at $x \in \partial \tilde{\omega}_{h}, \eta$ is the outward normal vector at $x \in \partial \omega_{h}$, and $\kappa_{\varphi}$ is the mean curvature of the level sets of $\varphi$.

It remains to estimate $I$. We again consider the two auxiliary functions, $\bar{u}$ and $v$ defined by (3.7). As before we have for $c:=\int_{\omega_{h}} u_{h}(\cdot, h)$ and $\bar{c}:=\int_{\tilde{\omega}_{h}} \bar{u}$ :

$$
\int_{\omega_{h}}\left|D u_{h}\right|^{2}(\cdot, h)-\int_{\tilde{\omega}_{h}}|D \tilde{u}|^{2}=\frac{c}{\bar{c}} \lambda\left(\int_{\tilde{\omega}_{h}} \bar{u}-\int_{\omega_{h}} u_{h}(\cdot, h)\right) .
$$

But this time the inequality $(\min [\bar{u}, \varphi(\cdot, h)]-v)_{+} \geq u_{h}(\cdot, h)$ holds on $\omega_{h}$, as

$$
\omega_{h}=\operatorname{supp}(\min [\bar{u}, \varphi(\cdot, h)]-v)_{+}
$$

and

$$
-\Delta(\min [\bar{u}, \varphi(\cdot, h)]-v) \geq \lambda .
$$

For the same reason we have for the outward normal $\eta$ of $\tilde{\omega}_{h}$

$$
\partial_{\eta}\left(u_{h}(\cdot, h)+v\right) \geq \partial_{\eta} \varphi(\cdot, h) .
$$

Thus, as $\min (\bar{u}, \varphi)=\bar{u}$ in $\tilde{\omega}_{h}$ and $\min (\bar{u}, \varphi)=\varphi$ in $\tilde{\omega}_{h} \Delta \omega_{h}$, using the smoothness of $\varphi$ it follows that

$$
\begin{aligned}
& \lambda\left(\int_{\tilde{\omega}_{h}} \bar{u}-\int_{\omega_{h}} u_{h}(\cdot, h)\right) \geq \lambda \int_{\tilde{\omega}_{h}} v-\lambda \int_{\omega_{h} \Delta \tilde{\omega}_{h}} \varphi(\cdot, h) \\
& \geq-\int_{\tilde{\omega}_{h}} \Delta\left(u_{h}(\cdot, h)+v\right) v+\int_{\omega_{h} \Delta \tilde{\omega}_{h}}(\Delta \varphi \varphi)(\cdot, h) \\
& +\int_{\omega_{h} \Delta \tilde{\omega}_{h}}(-\Delta \varphi-\lambda) \varphi(\cdot, h) \\
& \geq \int_{\tilde{\omega}_{h}}^{\omega_{h} \Delta \tilde{\omega}_{h}}\left(D\left(u_{h}(\cdot, h)+v\right)\right) D v-\int_{\partial \tilde{\omega}_{h}} \partial_{\eta}\left(u_{h}(\cdot, h)+v\right) v \\
& -\int_{\omega_{h} \Delta \tilde{\omega}_{h}}|D \varphi|^{2}(\cdot, h)+\int_{\partial \tilde{\omega}_{h}}\left(\partial_{\eta} \varphi \varphi\right)(\cdot, h)+\int_{\omega_{h} \Delta \tilde{\omega}_{h}} o(h) \\
& \geq \int_{\omega_{h}}|D v|^{2}-\int_{\omega_{h} \Delta \tilde{\omega}_{h}}|D \varphi|^{2}(\cdot, h)+\int_{\omega_{h} \Delta \tilde{\omega}_{h}} o(h)
\end{aligned}
$$

by (3.14) and (3.7). Thus together with (3.13) we have

$$
I \geq \frac{c}{\bar{c}} \int_{\omega_{h} \Delta \tilde{\omega}_{h}}|D \varphi|^{2} .
$$

Lastly we need to show that

$$
\bar{c} \rightarrow c \text { as } h \rightarrow 0 .
$$

To see this, first note that $u_{h}(x, h) \leq(\varphi(x, h)+h)_{+}$. In particular

$$
u_{h}(\cdot, h) \leq C h \quad \text { on } \partial \tilde{w}_{h}-\partial w_{h} \subset \partial\{x: \varphi(x, h)+h \geq 0\}
$$


where $C$ depends on the $C^{2}$-norm of $\varphi$. It follows that $\left.u_{h}(\cdot, h)\right|_{\omega_{h}} \leq \bar{u}_{h}+C h$, and therefore $c \leq \bar{c}+O(h)$. Hence we conclude.

\section{The continuum limit and existence of weak solutions}

In this section we show that in the limit $h \rightarrow 0$ and $\epsilon=h$ the time discrete solution $u_{h}$ converges to a weak solution $u(\cdot, t) \in H^{1}(B)$ of $(P)$ in the sense that the liminf and limsup -envelopes satisfy the barrier property at infinitesimal time scale (see Definition 4.4). We begin by defining viscosity sub- and supersolutions for a given multiplier function $\lambda(x, t)$ : $B \times[0, \infty) \rightarrow[0, \infty)$.

Definition 4.1 A lower semi-continuous function $u: B \times[0, \infty) \rightarrow \mathbb{R}$ is a viscosity supersolution on $\left[t_{1}, t_{2}\right]$ with respect to $\lambda(x, t)$ if following holds:

For a given function $\phi \in C^{2,1}(\overline{\{\phi>0\}})$ with $|D \phi| \neq 0$ in $B_{r}\left(x_{0}\right) \times\left[t_{1}, t_{2}\right]$, suppose that $\phi \leq u$ on the parabolic boundary of $B_{r}\left(x_{0}\right) \times\left[t_{1}, t_{2}\right]$ with

$$
\begin{array}{r}
-\Delta \phi(\cdot, t)<\lambda(\cdot, t) \quad \text { in } B_{r}\left(x_{0}\right) \times\left[t_{1}, t_{2}\right], \\
\frac{\phi_{t}}{|D \phi|}-\left(|D \phi|^{2}-1\right)<0 \quad \text { in } \Gamma(\phi) \cap\left\{B_{r}\left(x_{0}\right) \times\left[t_{1}, t_{2}\right]\right\} .
\end{array}
$$

Then $\phi \leq u$ in $B_{r}\left(x_{0}\right) \times\left[t_{1}, t_{2}\right]$.

For the subsolution part, in the context of our limit as $h \rightarrow 0$, we have to take into account the possibility that $\left\{u_{h}>0\right\}$ leave thin segments or isolated points in the limit, which are not traceable from the limit of $u_{h}$. We get around this difficulty by including a set $\Sigma$ in the definition:

Definition 4.2 Let $u: B \times[0, \infty) \rightarrow \mathbb{R}^{+}$be upper semi-continuous, and let $\Sigma$ be a closed subset of $B \times[0, \infty)$ containing $\Omega(u)$. Then the pair $(u, \Sigma)$ is a viscosity sub-solutionon $\left[t_{1}, t_{2}\right]$ with respect to $\lambda(x, t)$ if the following holds:

For a given function $\phi \in C^{2,1}(\overline{\{\phi>0\}})$ with $|D \phi| \neq 0$ in $B_{r}\left(x_{0}\right) \times\left[t_{1}, t_{2}\right]$, suppose that

$$
\begin{array}{r}
-\Delta \phi(\cdot, t)>\lambda(\cdot, t) \quad \text { in } B_{r}\left(x_{0}\right) \times\left[t_{1}, t_{2}\right], \\
\frac{\phi_{t}}{|D \phi|}-\left(|D \phi|^{2}-1\right)>0 \quad \text { in } \Gamma(\phi) \cap\left\{B_{r}\left(x_{0}\right) \times\left[t_{1}, t_{2}\right]\right\} .
\end{array}
$$

If $u \leq \phi$ and $\Sigma \subset \Omega(\phi)$ on the parabolic boundary of $B_{r}\left(x_{0}\right) \times\left[t_{1}, t_{2}\right]$, then $u \leq \phi$ and $\Sigma \subset \Omega(\phi)$ in $B_{r}\left(x_{0}\right) \times\left[t_{1}, t_{2}\right]$.

Let us go back to the time discrete solutions $u_{h}$. Define

$$
\mathcal{G}:=\left\{k 2^{-n}: k, n \in \mathbb{N}\right\} \quad \text { and } \quad h=h(n)=2^{-n}, n \in \mathbb{N} .
$$

Then $u_{h}$ is defined on grid times $t \in \mathcal{G}$ by (2.5), with the choice of $\epsilon=h$. Due to the Dirichlet energy bound, along a subsequence (depending on $t \in \mathcal{G}$ )

$$
u_{h}(\cdot, t) \rightarrow u(\cdot, t) \quad \text { weakly in } H^{1}\left(\mathbb{R}^{N}\right) \text { for each } t \in \mathcal{G} .
$$

We then choose a common subsequence of $h(n)$ such that (4.1) holds along the same sequence for each time. We obtain a weak form of convergence in the continuum limit $h \rightarrow 0$ along a subsequence. Unfortunately a stronger, point-wise convergence of $u_{h}$ cannot be obtained 
without extra regularity of $u_{h}$ such as equicontinuity in time. Instead we consider the limit infimum and supremum:

$$
u_{*}(x, t):=\lim _{r \rightarrow 0} \inf _{\{|x-y| \leq r,|s-t| \leq r, h \leq r\}} u_{h}(y, s)
$$

and

$$
u^{*}(x, t):=\lim _{r \rightarrow 0} \sup _{\{|x-y| \leq r,|s-t| \leq r, h \leq r\}} u_{h}(y, s) .
$$

Let us also define

$$
\Sigma:=\left\{(x, t) \mid \exists \text { a sequence }\left(x_{h}, t_{h}\right) \rightarrow(x, t) \text { such that } x_{h} \in \Omega\left(u_{h}\left(\cdot, t_{h}\right)\right)\right\} .
$$

Note that $\Sigma$ contains $\Omega\left(u^{*}\right)$. $\Sigma$ is a closed set, including "traces" of supports of $u_{h}(\cdot, t)$ which may degenerate into zero set of $u^{*}$ in the limit $h \rightarrow 0$. Let us denote

$$
\Sigma(s):=\Sigma \cap\{t=s\} .
$$

Next we define appropriate limits for the multipliers to be used for $u^{*}$ and $u_{*}$.

Definition 4.3 For $\omega \subset B$, let us define

$$
\lambda^{i n}(\omega, c):=\lim _{\delta \rightarrow 0} \lambda\left(u_{\omega_{\delta}, c}\right)
$$

and

$$
\lambda^{\text {out }}(\omega, c):=\lim _{\delta \rightarrow 0} \lambda\left(u_{\omega^{\delta}, c}\right),
$$

with $\omega^{\delta}:=\{x: d(x, \omega) \leq \delta\}$ and $\omega_{\delta}:=\left\{x: B_{\delta}(x) \subset \omega\right\}$.

Clearly $\lambda^{\text {in }} \geq \lambda^{\text {out }}$, as $\omega_{\delta} \subset \omega^{\delta}$. Now we are ready to define our weak solution:

Definition 4.4 For functions $u_{1}, u_{2}: B \times[0, \infty)$ and a closed set $\Sigma \subset B \times[0, \infty)$, the triple $\left(u_{1}, u_{2}, \Sigma\right)$ is a weak solution of $(P)$ if the following holds:

(a) $u_{1} \leq u_{2}$ and $\left\{u_{2}>0\right\} \subset \Sigma$;

(b) $u_{1}$ is a viscosity supersolution with respect to $\lambda_{1}(x, t):=\lambda^{\text {out }}\left(\omega, c_{1}\right)$, where $\omega$ is the connected component of $\Omega_{t}\left(u_{2}\right)$ containing $x$ and

$$
c_{1}:=\int_{\omega} u_{1}(\cdot, t) d x
$$

(c) $\left(u_{2}, \Sigma\right)$ is a viscosity subsolution with respect to $\lambda_{2}(x, t):=\lambda^{i n}\left(\omega, c_{2}\right)$, where $\omega$ is the connected component of $\Omega_{t}\left(u_{1}\right)$ containing $x$ and

$$
c_{2}:=\int_{D} u_{2}(\cdot, t) d x \text {, where } D \text { is the connected component of } \Omega_{t}\left(u_{2}\right) \text { containing } \omega .
$$

Roughly speaking, $\lambda_{1}$ and $\lambda_{2}$ are respectively the smallest and the largest possible value of the multiplier one can obtain by the lim sup and lim inf operation at a given point $(x, t)$. These definitions are tailored for $u_{1}=u_{*}$ and $u_{2}=u^{*}$.

Remark 4.5 (1) The set $\Sigma$ could be considered as the space-time limsup of the supports $\left\{u_{h}>0\right\}$. Such $\Sigma$ appears in the study of viscosity solutions when there are possibilities of nonuniqueness/fattening of the zero set (see for example [2]). 
(2) Classical solutions (i.e. $u \in C^{2,1}(\bar{\Omega}(u))$ with smooth $\Gamma(u)$ satisfying $(P)$ in the classical sense), if they exist, would be weak solutions of $(P)$ in our definition with $u_{1}=u_{2}=u$ and $\Sigma=\Omega(u)$.

In the rest of the section we will show the following:

Theorem 4.6 The triple $\left(u_{*}, u^{*}, \Sigma\right)$ defined in (4.2)-(4.4) is a weak solution of $(P)$.

Remark 4.7 (1) In [8] it was proven that starting from a star-shaped initial data, there is a unique star-shaped weak solution $(u, u, \Omega(u))$ of $(P)$ for a short time, and for global time with additional symmetries in the initial data. Short-time existence of any nature for general smooth initial data is an open problem.

(2) For free boundary problems which satisfy a comparison principle and which has a unique solution (which is generically the case for the mean-curvature flow or $(P)$ with fixed $\lambda$ ), the sub-solution would stay below the super-solution, which would then yield that $u^{*} \leq u_{*}$. This in turn yields $u^{*}=u_{*}$ and in particular the uniform convergence of $u_{h}$ to a weak solution readily follows. Unfortunately for us this line of argument cannot be applied since $(P)$ does not satisfy a comparison principle.

Proposition 4.8 Let us define $\lambda_{1}(x, t)$ and $\lambda_{2}(x, t)$ as in Definition 4.4 with $u_{1}=u_{*}$ and $u_{2}=u^{*}$.

(a) Suppose $(x, t) \in \Sigma$. There is $x_{h} \in \Omega_{t_{h}}\left(u_{h}\right)$ such that $\left(x_{h}, t_{h}\right) \rightarrow(x, t)$. Let $w^{*}$ be the connected component of $\Sigma(t)$ containing $x$ and let $w_{h}$ be the corresponding connected component containing $x_{h}$. Then

$$
\lambda_{1}(x, t) \leq \liminf _{h \rightarrow 0} \lambda\left(u_{h}(\cdot, t)\right)(x)
$$

(b) Suppose $(x, t) \in \bar{\Omega}\left(u_{*}\right)$. Then there is $x_{h} \in \Omega_{t_{h}}\left(u_{h}\right)$ such that $\left(x_{h}, t_{h}\right) \rightarrow(x, t)$. Let $w_{*}$ be the connected component of $\Omega\left(u_{*}\right)$ containing $x$, and let $w_{h}$ be the corresponding connected component containing $x_{h}$. Then

$$
\limsup _{h \rightarrow 0} \lambda\left(u_{h}(\cdot, t)\right)(x) \leq \lambda_{2}(x, t) .
$$

Proof To prove (a), first note that for fixed $\delta$ we have that $u_{h}\left(\cdot, t_{h}\right)$ converges uniformly to zero outside of $\omega^{*, \delta}:=\left\{x: d\left(x, \omega^{*}\right) \leq \delta\right\}$. Therefore, we can lower $u_{h}$ to its essential part: there exists $\varepsilon_{h} \rightarrow 0$ such that $\tilde{u}_{h}:=\left(u_{h}-\varepsilon_{h}\right)_{+}$satisfies $\Omega_{t}\left(\tilde{u}_{h}\right) \subset \omega^{*, \delta}$. Moreover we have, by definition of $c_{1}$,

$$
\liminf _{h \rightarrow 0} \int_{\omega^{*, \delta}} u_{h} d x \geq c_{1} .
$$

Therefore,

$$
\begin{aligned}
\lambda_{1}(x, t) & \leq \lambda\left(u_{\omega^{*, \delta}, c_{1}}(\cdot, t)\right)(x) \\
& \leq \liminf _{h \rightarrow 0} \frac{\int_{\omega^{*, \delta}}\left|D \tilde{u}_{h}\right|^{2}(\cdot, t) d x}{c_{1}} \\
& =\liminf _{h \rightarrow 0} \lambda\left(u_{h}(\cdot, t)\right)(x) .
\end{aligned}
$$

To prove (b), note that for any $\delta>0$, there exists $h_{0}<\delta$, such that $w_{\delta}:=\left\{x: B_{\delta}(x) \subset w_{*}\right\}$ is contained in $w_{h_{0}}$ : Suppose $\omega_{\delta} \not \subset \omega_{h}$ for some $\delta>0$ for any small $h>0$. Then there 
exists a sequence of points $x_{h}$ converging to a point $\bar{x}$ in $\omega_{\delta}$ such that $u_{h}\left(t_{h}, x_{h}\right)=0$. This is a contradiction to the fact $\omega_{\delta} \subset \Omega_{t}\left(u_{*}\right)$. Therefore $\omega_{\delta} \subset \omega_{h}$ at least for a sequence of $h$ converging to zero. Furthermore, by definition of $c_{2}$,

$$
\limsup _{h \rightarrow 0} \int_{\omega_{\delta}} u_{h}(\cdot, t) d x \leq c_{2} .
$$

and

$$
\limsup _{h \rightarrow 0} \lambda\left(u_{h}(\cdot, t)\right)(x) \leq \lim _{\delta \rightarrow 0} \lambda\left(u_{\omega_{\delta}, c_{2}}\right)(x) \leq \lambda_{2}(x, t) .
$$

Proof of Theorem 4.6 The proof carries over the barrier properties of the time discrete solutions.

We will only show that $u_{*}$ is a viscosity supersolution of $(P)$ with respect to $\lambda_{1}(x, t)$. The subsolution part can be shown via parallel arguments.

Suppose there exists a smooth function $\phi$ as in Definition 4.1 in $S:=B_{r}\left(x_{0}\right) \times\left[t_{1}, t_{0}\right]$ such that $\phi$ crosses $u_{*}$ from below at $\left(x_{0}, t_{0}\right)$.: i.e. $u_{*}-\phi$ has a minimum zero at $\left(x_{0}, t_{0}\right)$. By using $\tilde{\phi}(x, t):=\left(\phi(x, t)-\sigma\left(x-x_{0}\right)^{2}+\sigma\left(t-t_{0}\right)\right)_{+}$with small $\sigma>0$ if necessary, one may assume that the minimum is strict in $S$. Then for small $h>0$ the function $u_{h}-\phi$ also has a minimum at $\left(x_{h}, t_{h}\right)$ in $S$ with $\left(x_{h}, t_{h}\right) \rightarrow\left(x_{0}, t_{0}\right)$ as $h \rightarrow 0$. Since by Definition 4.1

$$
-\Delta \phi(x, t)<\lambda_{1}(x, t),
$$

we have that by Proposition 4.8(a) there exists $\delta>0$ such that

$$
-\Delta \phi<\lambda\left(u_{h}\right)\left(\cdot, t_{0}\right) \quad \text { in } B_{\delta}\left(x_{0}\right) \text { for } 0<h<\delta .
$$

The above inequality as well as the second inequality in Definition 4.1 yield that $\phi$ satisfies (3.1) for $h$ and $r$ sufficiently small. Hence Proposition 3.1, applied to $\phi$ and $u_{h}$ at $\left(x_{h}, t_{h}\right)$ in $S$, yields a contradiction.

Acknowledgements Natalie Grunewald was supported by the German Science Foundation, DFG, through fellowship GR 3391/1-1. Inwon Kim is supported by NSF DMS 0700732. The authors thank Karl Glasner for helpful discussions. Natalie Grunewald thanks UCLA for its hospitality during her stay in the last year, during which most of this paper was written.

Open Access This article is distributed under the terms of the Creative Commons Attribution Noncommercial License which permits any noncommercial use, distribution, and reproduction in any medium, provided the original author(s) and source are credited.

\section{References}

1. Almgren, F., Taylor, J.E., Wang, L.: Curvature driven flows, a variational approach. SIAM J. Control Optim. 31, 387-437 (1993)

2. Cardiliaguet, P., Ley, O.: On the energy of a flow arising in shape optimization. Interfaces Free Bound. 10, 221-241 (2008)

3. Chambolle, A.: An algorithm for Mean Curvature Motion. Interfaces Free Bound. 6, 195-218 (2004)

4. Esedoglu, S., Smereka, P.: A variational formulation for a level set representation of multiphase flow and area preserving curvature flow. Commun. Math. Sci. 6, 125-148 (2008)

5. Evans, L.C., Gariepy, R.F.: Measure Theory and Fine Properties of Functions. CRC-Press (1992)

6. Evans, L.C., Spruck, J.: Motion of level sets by mean curvature. J. Differential Geom. 33, 635-681 (1991)

7. Glasner, K.B.: A boundary integral formulation of quasi-steady fluid wetting. J. Comput. Phys. 207, 529-541 (2005) 
8. Glasner, K.B., Kim, I.C.: Global-time solutions for a model of contact line motion. Interfaces Free Bound. 11, 37-60 (2009)

9. Greenspan, H.P.: On the motion of a small viscous droplet that wets a surface. J. Fluid Mech. 84, 125143 (1978)

10. Hocking, L.M., Miksis, M.J.: Stability of a ridge of fluid. J. Fluid Mech. 247, 157-177 (1993)

11. Jordan, R., Kinderlehrer, D., Otto, F.: The variational formulation of the Fokker-Planck equation. SIAM J. Math. Anal. 29, 1-17 (1998)

12. Kim, I.C.: Uniqueness and existence result of Hele-Shaw and Stefan problem. Arch. Ration. Mech. Anal. 168, 299-328 (2003)

13. Luckhaus, S., Sturzenhecker, T.: Implicit time discretization for the mean curvature flow equation. Calc. Var. PDE 3, 253-271 (1995)

14. Petrosyan, A., Yip, A.: Nonuniqueness in a free boundary problem from combustion. J. Geom. Anal. 18, 1098-1126 (2008)

15. Tanner, L.: The spreading of silicone oil drops on horizontal surfaces. J. Phys. D 12, 1473-1484 (1979) 